\title{
Necropsy study of association between sudden death and cardiac enzymes
}

\author{
J Burns, C M Milroy, B Hulewicz, C R West, S M Walkley, N B Roberts
}

Sub-department of Forensic Pathology, University of

Liverpool

J Burns, C M Milroy, B Hulewicz

Department of Public Health, University of Liverpool

C R West

Department of Chemical Pathology, University of

Liverpool

S M Walkley,

N B Roberts

Correspondence to: Dr C M Milroy, Department of Forensic Pathology,

University of Sheffield,

Watery Street,

Sheffield S3 7ES

Accepted for publication 18 September 1991

\begin{abstract}
Aims: To determine if cardiac enzymes measured at necropsy could be used to predict early myocardial infarction.

Methods: Cardiac enzyme activities were measured in body fluids at necropsy. Coroners' necropsies were grouped by gross and microscopic findings into cases of definite myocardial infarction, cases with occlusive coronary artery atheroma but no identifiable myocardial infarction, and non-cardiac cases. Pericardial fluid, peripheral venous blood, and blood from the right atrium were collected. Total creatine phosphokinase, creatine phosphokinase isoenzymes, aspartate aminotransferase and hydroxybutarate dehydrogenase activities were measured and the results analysed by logistic regression.
\end{abstract}

Results: The values of creatine phosphokinase and its isoenzymes were raised in those who had died of cardiac disease and were most discriminatory. Cases of early myocardial infarction without evidence of infarction on routine histological examination could be identified from enzyme activities.

Conclusions: Measurement of cardiac enzymes in blood and pericardial fluid at

Table 1 Non-cardiac deaths $(n=29)$

\begin{tabular}{llll}
\hline Site and enzyme & Range $(I U / l)$ & Median & Interquartile range \\
\hline Right atrial blood: & & & \\
Total CPK & $418-74500$ & 18300 & $9000-38680$ \\
BB & $0-782$ & 0 & $0-9$ \\
MB & $0-16811$ & 2220 & $1217-6439$ \\
MM & $348-68316$ & 13542 & $7147-29712$ \\
BB \% & $0-7$ & 0 & $0-0 \cdot 75$ \\
MB \% & $0-38 \cdot 7$ & $16 \cdot 7$ & $10 \cdot 75-24 \cdot 45$ \\
MM \% & $61 \cdot 3-100$ & $83 \cdot 2$ & $75 \cdot 6-89 \cdot 3$ \\
HBD & $320-43600$ & 7410 & $2338-17275$ \\
AST & $170-10125$ & 1700 & $650-4363$ \\
& & & \\
Peripheral venous blood $:$ & $220-257000$ & 17850 & $7815-32575$ \\
Total CPK & $0-2325$ & 0 & $0-450 \cdot 5$ \\
BB & $0-8940$ & 664 & $200-1477$ \\
MB & $177-248776$ & 17750 & $7248-30686$ \\
MM & $0-10 \cdot 9$ & 0 & $0-3 \cdot 55$ \\
BB \% & $0-11 \cdot 7$ & $5 \cdot 4$ & $3 \cdot 65-7 \cdot 4$ \\
MB \% & $80 \cdot 5-100$ & $93 \cdot 7$ & $89 \cdot 3-96 \cdot 4$ \\
MM \% & $210-30000$ & 1400 & $625-4525$ \\
HBD & $38-6250$ & 500 & $250-1450$ \\
AST & & & \\
Pericardial fluid: & $122-100750$ & 18120 & $6630-46260$ \\
Total CPK & $0-1680$ & 0 & $0-151 \cdot 5$ \\
BB & $0-27513$ & 2443 & $658-4344$ \\
MB & $77-97728$ & 17178 & $5814-38370$ \\
MM & $0-13 \cdot 1$ & 0 & $0-1 \cdot 7$ \\
BB \% & $0-31 \cdot 3$ & $14 \cdot 1$ & $6 \cdot 6-19 \cdot 1$ \\
MB \% & $63 \cdot 3-100$ & $85 \cdot 8$ & $78 \cdot 1-93 \cdot 4$ \\
MM \% & $175-23300$ & 2985 & $1318-7435$ \\
HBD & $19-7700$ & 550 & $238-1425$ \\
AST & & & \\
\hline & & & \\
\hline
\end{tabular}

necropsy can provide valuable additional information in cases of sudden death as a result of myocardial ischaemia which have occurred before macroscopic or microscopic evidence of myocardial infarction.

Sudden death from cardiac disease is the most common cause of death identified in necropsies performed for the Coroner. Identifiable myocardial infarction is not uncommon, but in most post mortem examinations severe occlusive coronary artery atheroma, without macroscopic or microscopic evidence of myocardial infarction, is the most common finding. ${ }^{1}$ Various techniques have been used to try to identify infarction microscopically earlier than can be seen on haematoxylin and eosin staining but these are not regularly used because of various difficulties including ease of use and variability of results. $^{2-13}$

\section{Methods}

Necropsies performed for the Coroner were grouped into non-cardiac deaths, deaths from occlusive coronary artery atheroma without evidence of infarction, and deaths from definite myocardial infarction.

Multiple blocks of paraffin wax embedded myocardium, stained with haematoxylin and eosin, were examined to identify or exclude infarction. Blood from the right atrium (into which the coronary sinus drains), blood from a peripheral vein, and pericardial fluid were collected, and cardiac enzyme activities measured in each. The following enzymes were measured: aspartate aminotransferase (AST); hydroxybutarate dehydrogenase (HBD); total creatine phosphokinase (CPK); creatine phosphokinase isoenzymes CPK-MM, CPK-MB, and CPK-BB.

The creatine phosphokinase isoenzymes were measured by quantitative electrophoresis (Ciba-Corning, Palo-Alto, California, USA). Enzyme activity was measured by a standard procedure using a centrifugal analyser (Multistat IL, Warrington, England). Total CPK on each sample was measured, then a second sample with activity up to $1000 \mathrm{IU} / 1$ applied for electrophoresis. Samples with activity greater than $1000 \mathrm{IU} / 1$ were diluted using isotonic saline accordingly.

A total of 72 cases were collected in which full data were included, and in 66 of these cases the interval between death and necropsy was also known (the post mortem examination interval). 
Results

From the results of the necropsy combined with the histological evidence, 29 of the 72 cases were classified as non-cardiac deaths, 15 as definite myocardial infarction, and 28 as

Table 2 Possible infarct $(n=28)$

\begin{tabular}{|c|c|c|c|}
\hline Site and enzyme & Range (IU/l) & Median & Interquartile range \\
\hline $\begin{array}{l}\text { Right atrial blood: } \\
\text { Total CPK } \\
\text { BB } \\
\text { MB } \\
\text { MM } \\
\text { BB \% } \\
\text { MB \% } \\
\text { MM \% } \\
\text { HBD } \\
\text { AST }\end{array}$ & $\begin{array}{l}1550-75700 \\
0-2102 \\
281-13626 \\
1202-62074 \\
0-4 \cdot 8 \\
6 \cdot 4-29 \cdot 9 \\
68 \cdot 2-93 \cdot 6 \\
1050-24400 \\
300-5600\end{array}$ & $\begin{array}{l}16825 \\
0 \\
3081 \\
13263 \\
0 \\
16 \cdot 85 \\
83 \cdot 15 \\
4900 \\
1950\end{array}$ & $\begin{array}{l}9125-29950 \\
0-160 \\
1247-5159 \\
7924-25445 \\
0-1 \cdot 5 \\
6 \cdot 4-29 \cdot 9 \\
76 \cdot 2-87 \cdot 2 \\
2680-10720 \\
875-3788\end{array}$ \\
\hline $\begin{array}{l}\text { Peripheral venous blood: } \\
\text { Total CPK } \\
\text { BB } \\
\text { MB } \\
\text { MM } \\
\text { BB \% } \\
\text { MB \% } \\
\text { MM \% } \\
\text { HBD } \\
\text { AST }\end{array}$ & $\begin{array}{l}469-150750 \\
0-5427 \\
0-7326 \\
411-138087 \\
0-6 \cdot 1 \\
0-17 \cdot 5 \\
80 \cdot 5-100 \\
230-16900 \\
93-5125\end{array}$ & $\begin{array}{l}16575 \\
0 \\
556 \\
15062 \\
0 \\
4 \cdot 75 \\
93 \cdot 1 \\
1350 \\
450\end{array}$ & $\begin{array}{l}3688-54345 \\
0-136 \cdot 5 \\
145-2391 \\
3536-50788 \\
0-3 \cdot 3 \\
2 \cdot 76-7 \cdot 0 \\
89 \cdot 9-97 \cdot 4 \\
825-3038 \\
250-1050\end{array}$ \\
\hline $\begin{array}{l}\text { Pericardial fluid: } \\
\text { Total CPK } \\
\text { BB } \\
\text { MB } \\
\text { MM } \\
\text { BB \% } \\
\text { MB \% } \\
\text { MM \% } \\
\text { HBD } \\
\text { AST }\end{array}$ & $\begin{array}{l}6300-135000 \\
0-983 \\
0-25342 \\
5218-13500 \\
0-1 \cdot 4 \\
0-36 \cdot 1 \\
62 \cdot 4-100 \\
1300-28700 \\
250-7850\end{array}$ & $\begin{array}{l}24320 \\
0 \\
1955 \\
21114 \\
0 \\
10 \cdot 5 \\
89 \cdot 6 \\
4075 \\
950\end{array}$ & $\begin{array}{l}13700-55400 \\
0-0 \\
941-3290 \\
12791-49135 \\
0-0 \\
5 \cdot 2-14 \cdot 5 \\
85 \cdot 5-94 \cdot 8 \\
2047-6700 \\
650-2350\end{array}$ \\
\hline
\end{tabular}

Table 3 Definite infarction $(n=15)$

\begin{tabular}{llll}
\hline Site and enzyme & Range $(I U / l)$ & Median & Interquartile range \\
\hline Right atrial blood: & & & \\
Total CPK & $6250-112600$ & 39120 & $9200-76200$ \\
BB & $0-641$ & 0 & $0-148 \cdot 8$ \\
MB & $635-13878$ & 4765 & $1394-7672$ \\
MM & $4867-106069$ & 32154 & $7288-53085$ \\
BB\% & $0-7$ & 0 & $0-2 \cdot 1$ \\
MB \% & $3 \cdot 7-23 \cdot 1$ & $15 \cdot 2$ & $11 \cdot 7-17 \cdot 9$ \\
MM \% & $75 \cdot 0-96 \cdot 3$ & $83 \cdot 4$ & $79 \cdot 9-88 \cdot 3$ \\
HBD & $1650-28550$ & 9713 & $4863-16013$ \\
AST & $200-9275$ & 2263 & $1344-5130$ \\
Peripheral venous blood: & & & \\
Total CPK & $2160-203800$ & 13350 & $4838-40470$ \\
BB & $0-2252$ & 109 & $0-260$ \\
MB & $0-4170$ & 467 & $212-1206$ \\
MM & $2029-203800$ & 12401 & $4340-39072$ \\
BB \% & $0-5 \cdot 6$ & $2 \cdot 1$ & $0-4 \cdot 7$ \\
MB \% & $0-6 \cdot 4$ & $4 \cdot 8$ & $3 \cdot 8-5 \cdot 8$ \\
MM \% & $88 \cdot 2-100$ & $93 \cdot 4$ & $90 \cdot 0-96 \cdot 2$ \\
HBD & $335-31400$ & 2800 & $850-7800$ \\
AST & $100-8275$ & 775 & $250-3500$ \\
Pericardial fluid: & & & \\
Total CPK & $1390-215400$ & 16400 & $6920-58500$ \\
BB & $0-571$ & 0 & $0-0$ \\
MB & $97-38183$ & 1288 & $607-4344$ \\
MM & $1293-184813$ & 15793 & $6076-53293$ \\
BB\% & $0-10 \cdot 8$ & 0 & $0-0$ \\
MB \% & $3 \cdot 7-28 \cdot 2$ & $10 \cdot 3$ & $7 \cdot 9-12 \cdot 7$ \\
MM \% & $71 \cdot 8-96 \cdot 3$ & $89 \cdot 1$ & $85 \cdot 8-92 \cdot 1$ \\
HBD & $189-40100$ & 650 & $425-2575$ \\
AST & $50-7800$ & & \\
\hline & & 3700 & \\
\hline
\end{tabular}

Table 4 Model using total CPK enzymes and isoenzyme percentages

\begin{tabular}{|c|c|c|}
\hline \multicolumn{3}{|c|}{$\begin{aligned} \text { Logit }(p)=1.120 & +1.438 \log \text { (total right atrial CPK) } \\
& -0.040(\% \text { isoenzyme } M B \text { in peripheral venous blood }) \\
& =0.130(\% \text { isoenzyme } M B \text { in pericardial fluid }) \\
& -0.072(\% \text { isoenzyme } M M \text { in pericardial fluid })\end{aligned}$} \\
\hline $\begin{array}{l}\text { Classification } \\
\text { Non-cardiac } \\
\text { Infarct } \\
\text { Possible infarct }\end{array}$ & $\begin{array}{l}\text { Predicted non-infarct } \\
25 \\
9 \\
24\end{array}$ & $\begin{array}{l}\text { Predicted infarct } \\
4 \\
6 \\
4\end{array}$ \\
\hline
\end{tabular}

possible myocardial infarction (occlusive coronary artery atheroma only).

The enzyme activities varied widely (tables 1-3) and there was a steady rise in enzyme activities the longer the interval before post mortem examination. The percentage of isoenzymes, however, remained fairly constant. The post mortem examination interval varied from two to 99 hours (mean 31.8 hours, median 21 hours).

The results were statistically analysed using logistic regression and discriminant analysis. ${ }^{14}{ }^{15}$ Both logistic regression and discriminant analysis gave similar results. Logistic regression is the preferred technique, because it does not assume multivariate normality of the predictor variables and leads to a direct estimate of probability of death from cardiac cause without resorting to Bayes' theorem.

Using logistic regression, $L=\operatorname{logit}(p)=\ln$ $(p /[1-p])$, whence $p=\exp (L) / 1+\exp (L) \cdot p$ is the estimated probability of the death being due to myocardial infarction, and "predicted positive" means $p>0.5$ (which is the same as $L$ $>0$ ); two models were found to be the best predictors.

Using a combination of total CPK in the right atrial blood together with percentage isoenzyme $\mathrm{MB}$ in peripheral venous blood, percentage isoenzyme MB in pericardial fluid, and percentage isoenzyme $M M$ in pericardial fluid, the results in table 4 were obtained. When the post mortem examination interval was known and included in the analysis, a better rate of classification was obtained (table $5)$. In this model, about $75 \%$ of cases of myocardial infarction and non-cardiac deaths would be correctly classified according to enzyme results. Ten of the 27 cases of possible myocardial infarction (the occlusive coronary artery atheroma group) would be reclassified as myocardial infarction. Hydroxybutyric dehydrogenase and aspartate aminotransferase did not provide any additional independent information.

Activities of CPK-BB up to $5 \%$ were found in pericardial fluid in several cases with a variety of causes of death which included occlusive coronary artery atheroma (3), bronchopneumonia (6), lobar pneumonia (1) cerebral haemorrhage (1), asphyxia following choking on food (1), hypothermia (1), pancreatitis (1), congestive cardiac failure (1) and gastrointestinal haemorrhage (1).

Higher CPK-BB values in pericardial fluid were seen in cerebral infarction $(39.8 \%)$, cerebral damage caused by lithium toxicity $(13 \cdot 1 \%)$, and epilepsy $(6 \cdot 4 \%)$.

\section{Discussion}

Reliable markers of early myocardial infarction still elude pathologists. Early histological changes of myocardial infarction seen on haematoxylin and eosin stained sections include oedema, eosinophilia, and granularity of fibres. These changes can also be produced by autolysis and variation in section thickness 
Table 5 Model using post-mortem examination interval, total CPK, and percentage isoenzymes

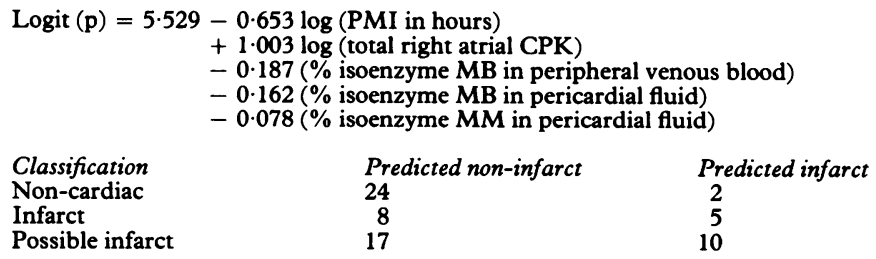

Classification
Non-cardiac

Infarct

Possible infarct

$\begin{array}{lc}\text { Predicted non-infarct } & \text { Predicted infarct } \\ 24 & 2 \\ 8 & 5 \\ 17 & 10\end{array}$

and staining, all of which make accurate determination of early changes difficult. Similarly, waviness of fibres, another early change, may be seen in the absence of infarction.

The factors which make early recognition of myocardial infarction unreliable have prompted searches for a more reliable method of detection of early myocardial infarction. Such techniques have included staining of paraffin wax sections with haematoxylin-basic fuschinpicric acid, acridine orange (and subsequent examination under ultraviolet light) and enzyme histochemistry using frozen sections. These methods have either proved unreliable or difficult to perform. ${ }^{2-13}$

Measurement of cardiac enzymes in pericardial fluid at necropsy, but not in blood, have been undertaken by Luna and colleagues, ${ }^{1617}$ and by Stewart and his colleagues. ${ }^{18}$ In their first paper Luna et al measured total LDH and its isoenzymes and total CPK in a variety of cases at necropsy. They found raised total CPK values in people dying from myocardial infarction, violent asphyxia, and multiple trauma compared with deaths from pulmonary embolism, infectious bronchopulmonary disease, haemorrhage and trauma of the central nervous system and a group labelled miscellaneous deaths. Total LDH values were not discriminatory, but the $\mathrm{LDH}_{1}: \mathrm{LDH}_{2}$ isoenzyme ratio was increased in those who had died from myocardial infarction. In a second study Luna et al found increased CPK-MB values in those who had died from myocardial infarction compared with deaths from other causes. They did not find any significant difference between values in myocardial infarction and cases of multiple trauma. In neither study did they differentiate between cases of definite myocardial infarction and occlusive coronary artery atheroma.

Stewart et al measured creatine phosphokinase, lactate dehydrogenase and their isoenzyme activities in pericardial fluid at necropsy. They found raised cardiac enzyme activities in cases of cardiac death compared with non-cardiac deaths. Enzyme activities were not statistically different between those cardiac cases with histological evidence of infarction and those without (occlusive coronary artery atheroma only). Values were raised in people where resuscitation had been attempted before death, and like Luna et al, they found raised activities in those who had died a violent death, defined by Stewart $e t$ al as a death in which there was or should have been severe physical or emotional distress. The exact cause of death in these cases of violent death was not defined but, interestin- gly, they had the highest values of any group, with no evidence of myocardial necrosis. This was attributed to the action of catecholamines ("stress" cardiomyopathy).

Luna et al did not find CPK-BB in pericardial fluid in any of their cases, but Stewart et al did in two cases with central nervous system pathology. We found high CPK-BB activities in pericardial fluid in cases with intracranial disease, as well as low values in cases with various causes of death.

Our study has shown that death caused by early myocardial infarction can be predicted from cardiac enzyme activities at necropsy. Most non-cardiac deaths were correctly predicted (24 out of 26 ) using the model in table 5 . Five of the 13 cases in which there was histological evidence of infarction were predicted as cardiac deaths using this model. The fact that eight cases were not is not entirely surprising as the rise in enzymes may have occurred a few days before death and then returned to normal. In the cases of possible myocardial infarction 10 cases were classified as infarction, based on the enzyme activities. In these cases the rise in enzymes suggests that infarction was occurring but that death happened before histological evidence of infarction appeared. In the other 17 cases there was no histological or biochemical evidence of infarction. This is not surprising. In many of these cases there was probably a cardiac arrhythmia and very rapid death without any infarction. Very early infarction, with no rise in enzymes, or an insignificant rise, however, cannot be excluded.

The results of this study show that early myocardial infarction can be identified at necropsy using cardiac enzyme measurement of body fluids. The accuracy is increased if blood from the right atrium and peripheral venous blood is used in addition to pericardial fluid. If the post mortem examination interval is included in the analysis the accuracy is increased still further. The failure to identify a rise in enzymes in all cases of the definite infarction group does not, of course, present any practical problem, as infarction will be identified by gross or routine histological examination. Although not all cases of early myocardial infarction can be predicted using our model, any additional information when only occlusive coronary artery atheroma is present may be useful in controversial cases. We recommend, therefore, in cases of sudden unexpected death, when post mortem examination indicates myocardial ischaemia as the likely cause of death, but where doubt still exists, that pathologists make use of this valuable additional information by taking samples of blood and pericardial fluid for enzyme analysis.

This study was supported by a $\mathrm{CH}$ Milburn research grant from the British Medical Association.

We thank Joan Holtom for her technical assistance.

1 Knight B. In: The pathology of sudden death. Forensic pathology. London: Edward Arnold, 1991:444-54.

2 McVie JG. Postmortem detection of inapparent myocardial infarction. Pathology 1970;23:203-9.

3 Lie JT, Holley KE, Kampa WR, Titus JL. New histochemical methods for morphologic diagnosis of early 
stages of myocardial ischaemia. Mayo Clin Proc 1971; 46:319-27.

4 Bouchardy B, Manjo G. Histopathology of early myocardial infarcts. Am J Pathol 1974;74:301-17.

5 Rammer L, Jannsen O. Determination of electrolytes in the myocardium as a tool for the postmortal diagnosis of recent infarction. Forensic Sci 1976;8:127-30.

6 Sahai VB, Knight BH. The post-mortem detection of early myocardial infarction by a simple fluorescent method. Med Sci Law 1976;16:17-20.

$7 \mathrm{Knight}$ BH. A further evaluation of the reliability of the HBFP stain in demonstrating early myocardial damage. Forensic Sci 1979;13:179-81.

8 Pedersen PK. Determination of potassium/sodium ratio in heart tissue. Evaluation of its use as an index of myocardial ischaemic damage. Forensic Sci Int 1980;16:272-80.

9 Derias NW, Adams CW. The non-specific nature of the wavy myocardial fibre. Histopathology 1979;3:241-5.

10 Derias NW, Adams CW. Macroscopic enzyme histochemistry in myocardial infarction: use of enzyme histochemisphenazine methosulphate. J Clin Pathol 1982;35:410-3.

11 Badir B, Knight B. Fluorescence microscopy in the detection of early myocardial infarction. Forensic Sci Int 1987;
34:99-106.

12 Varga $M$, Zsonda L. A simple method for the postmortem detection of acute myocardial infarction. Forensic Sci Int 1988;37:259-63.

13 Lachia E, Villaneuva E, Luna A. Comparison of different techniques for the postmortem diagnosis of myocardial infarction. Forensic Sci Int 1988;38:21-6.

14 Altman DG. In: Relation between several variables. Practical statistics for medical research. London: Chapman Hall, 1991:351-8.

15 Altman DG. In: Relation between several variables. Practical statistics for medical research. London: Chapman Hall, 1991:358-60.

16 Luna A, Villanueva E, Castellano MA, Jimenez G. The determination of CK, LDH and its isoenzymes in pericardial fluid and its application to the post-mortem diagnosis of myocardial infarction. Forensic Sci Int 1982;19:85-91.

17 Luna A, Carmona A, Villanueva E. The postmortem determination of CK isoenzymes in pericardial fluid in various mination of CK isoenzymes in pericardial fluid in

18 Stewart RV, Zumalt RE, Hirsch CS, Kaplan L. Postmortem diagnosis of myocardial disease by enzyme analysis of pericardial fluid. Am J Clin Pathol 1984;82:411-7.

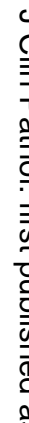

